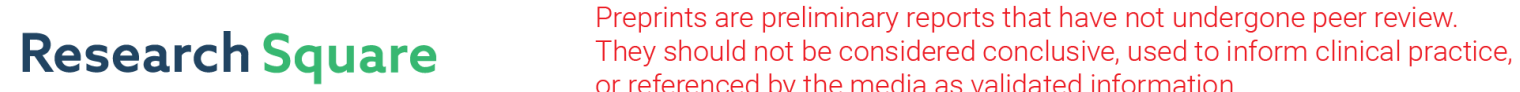 or referenced by the media as validated information. \\ Persuasive Messaging to Encourage COVID-19 Risk Reduction
}

Scott Bokemper ( $\nabla$ scott.bokemper@yale.edu )

Yale University https://orcid.org/0000-0002-9754-4658

\section{Gregory Huber}

Yale University https://orcid.org/0000-0001-6804-8148

\section{Erin James}

Yale Institute for Global Health

\section{Alan Gerber}

Yale University

\section{Saad Omer}

Yale University https://orcid.org/0000-0002-5383-3474

\section{Article}

Keywords: public health messages, COVID-19, public health campaign

Posted Date: October 28th, 2020

DOI: https://doi.org/10.21203/rs.3.rs-96261/v1

License: (c) (1) This work is licensed under a Creative Commons Attribution 4.0 International License. Read Full License 


\section{Abstract}

What types of public health messages are effective at changing people's beliefs and intentions to practice social distancing to slow the spread of COVID-19? We conducted two randomized experiments that assigned respondents to read a public health message and then measured their beliefs and behavioral intentions across a wide variety of outcomes. Using both a convenience sample and a nationally representative sample of Americans, we find that a message that reframes bravery as recklessness and a message that highlights the need for everyone to take action to protect others are the most effective at increasing beliefs and intentions related to social distancing. These results provide an evidentiary basis for building effective public health campaigns to increase social distancing during flu pandemics.

\section{Main Text}

Governments and public health officials have emphasized the importance of social (physical) distancing and other related measures in mitigating the spread of COVID-19. Given the present lack of an effective vaccine and the limitations of available post-infection medical treatments, the need for interventions that cause individuals to take actions that reduces the risk of infection has become increasingly important. In practice, many messaging and communication strategies have been observed. However, despite these widespread and varied efforts, we lack an evidentiary basis for understanding the messages that are effective at increasing individuals' willingness to embrace actions that reduce the spread of COVID-19 without also causing unintended consequences like depressing their willingness to get a future vaccine or participating in elections.

Existing work on public health messaging has demonstrated behavioral change in response to specific messages about tobacco use, consumption of sugary beverages, high risk sexual behavior, and vaccination take-up (1-5). Messages used in past work often target one or a very small number of behaviors at a time.

However, successful public health strategies that address the COVID-19 pandemic require large numbers of people to change a broad range of daily behaviors, such as how they interact with friends and relatives, whether they wear face coverings in public, and cooperation with government efforts to identify infectious individuals. This suggests that a more fruitful messaging strategy needs to change attitudes towards social distancing more broadly rather than targeted messaging to increase the prevalence of a specific action. Changes in attitudes could also increase the willingness of individuals to encourage others to engage in these behaviors - that is, to reinforce desired behaviors through social norms. Importantly, unlike other health behaviors, many individuals are at a relatively low risk of serious COVID19 complications, but their behavior is nonetheless important for reducing the risk to individuals who are more vulnerable as the disease continues to spread throughout the general population.

We conducted two experiments to examine how different public health messages affect people's beliefs about the efficacy of social distancing, their intentions to practice social distancing, and their attitudes 
about enforcing social norms, such as persuading others to practice social distancing and negatively judging those who do not. We also test whether messaging that is designed to encourage people to practice social distancing has unintended consequences such as discouraging people from going to vote in-person or receiving a vaccine were it to become available.

In Experiment 1, we test the efficacy of a large number of messages against a baseline control message that describes social distancing. In Experiment 2, we take the two most successful messages from Experiment 1 and conduct a registered trial using a nationally-representative sample of American adults against both an informational control and a placebo-treated control group. In our second study, we also examined mask wearing and the willingness to self-isolate if exposed to COVID-19 as well as cooperate in contact tracing. In both studies, we examine the possibility that certain messages are more effective among specific segments of the population.

Experiment 1 was conducted in late May 2020. Participants were randomly assigned to read a baseline message that defined social distancing and stated that public health experts believe it would reduce the spread of COVID-19 or to one of ten treatment messages grouped into three categories. Each treatment message was added to the baseline message. The first category of messages stated that people who believe they are being brave by continuing with their daily routines despite the threat of the virus are actually being reckless. That is, it takes a desirable value and reframes it as selfish and unattractive ("people who don't practice social distancing... aren't brave, they are reckless"). Bravery and risk-taking are viewed as attractive traits across a variety of cultures (6-8). This reframing was either presented alone (Not Bravery) or with language about how people who spread COVID-19 pollute the environment around them (Not Bravery + Pollution).

The second category of messages varied the beneficiary of social distancing behaviors and whether individual or collective action was needed to produce these benefits. The beneficiary of social distancing was either the individual ("you could get sick and die") or others ("members of your family and community could get sick and die"). Past work has examined the effectiveness of self-interested frames compared to cooperative frames in public health messaging (9-11). We combined manipulation of the beneficiary with what was necessary to produce this benefit. Specifically, social distancing was framed as providing a benefit if an individual practiced it (individual action, "when you practice social distancing you reduce the risk"), if enough other people practiced it (threshold collective action, "if enough people practice social distancing then we can reduce the risk"), or for each additional person who practiced (linear collective action, "every person who practices social distancing reduces the risk"). The latter two frames were designed to tap into beliefs about social norms and the psychology of cooperative public goods production (12-16). Crossing these two dimensions of manipulation produced the six total treatment messages in this category.

The final category of messages invoked the idea that practicing social distancing would facilitate returning to "normal" life before the COVID-19 pandemic ("Social distancing now means we can more quickly return to our normal way of life") or that doing so involved adapting to an unavoidable "new 
normal" ("we are adapting to the 'new normal' necessary because of COVID-19"). These two messages were designed to mirror rhetoric being used by political leaders and in the media.

We form four mean scales as outcome measures, with all scales ranging from 0 to 1 with 1 indicating behaviors or beliefs associated with reducing the spread of COVID-19 (see Supplementary Materials for question wording and scale distributions). The four scales were: 1) a BELIEFS/norms scale that assesses agreement with beliefs about social distancing being important for your health and others people's health and whether an individual would feel guilty for not practicing social distancing, 2) a social distancing (OWN-SD) scale that captures people's intended willingness to social distance, avoid attending gatherings, forego elective medical procedures, and wear a mask, 3) a FOOD behavior scale that assesses people's willingness to avoid high-risk food related behavior like going to a restaurant, and 4) a persuade/evaluate OTHERS scale that measures whether people would persuade others to social distance, report a business for violating rules, and negatively judge non-compliers. We also asked respondents about their willingness to receive a potential COVID-19 vaccine if one were to become available and their interest in voting by mail or voting in person if that was their only option to assess unintended consequences of communication to encourage social distancing. Several items in the OWNSD and FOOD scales had previously been shown to be affected by rhetoric focusing on selfish and prosocial motivations for social distancing (10).

(Fig. 1 Here)

We analyze our data using OLS regression comparing outcomes to the informational baseline using indicators for each treatment and including pre-treatment demographic covariates to improve efficiency. Two messages appear particularly promising compared to the baseline message, with all treatment effect estimates plotted in Panel A of Fig. 1. (Underlying regression analysis appears in Table S1.) Among the messages that appear most effective, the Not Bravery message increases all four scale outcomes. The estimated effect on the OTHERS scale is .058 (95\% Cl: .023, .092), indicating that respondents who read the Not Bravery message report more willingness to enforce norms to promote social distancing. We also observe suggestive evidence that this message affects both the BELIEFS scale and the OWN-SD scale. For the BELIEFS scale the estimate is $.037(95 \% \mathrm{Cl}:-.005, .079)$ or about $12.8 \%$ of a standard deviation, while the effect for the OWN-SD scale is .030 ( $95 \% \mathrm{Cl}:-.004, .064)$ or about $13 \%$ of a standard deviation. The estimated effect for the FOOD scale is positive, but imprecise.

The Other-regarding, Linear Cooperation (ORLC) message also performs well relative to the baseline control condition. For all four scales, the estimated effects of this message are positive. For the OWN-SD scale, the effect is $.034(95 \% \mathrm{Cl}: .002, .067)$ or $14.7 \%$ of a standard deviation. Respondents' beliefs about the importance of social distancing also increase with an estimated effect of .040 (95\% Cl: $-.002, .084)$ on the BELIEFS scale. The effect on the FOOD scale is $.038(95 \% \mathrm{Cl}:-.003, .079)$. The latter two effects are not statistically significant at the conventional $5 \%$ level, but do provide evidence that the ORLC message broadly moved beliefs relevant to practicing social distancing. Notably, each of these two messages performs best of any message on two of the four scales. 
It is also interesting that two messages appear, on average, less effective than the baseline content to which they are added. While no coefficient estimates are individually statistically significant, both the Return to Normal and New Normal messages are generally less effective than their baseline content across our primary outcome measures.

Policymakers have also expressed interest in learning if concerns about COVID-19 are likely to have effects on voting in the 2020 fall elections and a willingness to take-up the COVID-19 vaccine if it becomes available. We report identical analysis for three ancillary outcomes in Panel B of Fig. 1. Reassuringly, the two messages that were most effective on average did not suppress interest in voting in person if necessary and may increase (not significant) interest in voting by mail. There is no evidence that the Not Bravery message or ORLC message decreases willingness to take up a COVID-19 vaccine in the future.

We also conduct a number of exploratory analyses for heterogeneous treatment effects by age, gender, partisanship, and geographic location and do not uncover large differences in treatment effectiveness across these groups (Figures S2-S6). Due to the rhetoric among the public and political elites surrounding the degree to which measures to address the spread of COVID-19 infringe upon people's liberties, we elicited people's adoption of a liberty moral foundation that captures their belief about the role of government in society (17). We found evidence that intervention effectiveness varies by endorsement of liberty values. Compared to respondents below the mean in their adoption of liberty values, respondents who are above the mean in their adoption of liberty are more responsive to the Not Bravery message than to the baseline control condition on the BELIEFS scale $(p=.05)$ and OTHERS scale $(p<.01)$, with weaker evidence for the OWN-SD scale $(p=.14)$. The effects of the Not Bravery message are uniformly statistically insignificant for those low in liberty (See Figure S6).

Experiment 2 tests the two most successful treatments in Experiment 1 (Not Bravery, and ORLC) and the Baseline compared to a Control message about an unrelated topic (Bird feeding). Experiment 2 is a preregistered trial fielded between mid-July and early August 2020, a time when the COVID-19 outbreak in the United States had become far more widespread than during Experiment 1. We made incremental changes to the four scales (BELIEFS, OWN-SD, FOOD, and OTHERS) used in Experiment 1 to reflect changing policies and circumstances, most importantly adding items to the OWN-SD scale about attendance at religious services, participation in political events, self-isolation following COVID-19 exposure, and alerting public health authorities if diagnosed with COVID-19, and for the OTHERS scale adding an item about cooperating in contact tracing. We also included a MASK scale composed of items about wearing a face covering in six circumstances, as well as relative willingness to shop at a store that requires rather than prohibits face masks. We again test the effect for our secondary outcomes to capture any unintended consequences.

At the time this experiment was fielded, messaging outside of the experimental context on the importance of OWN-SD had become far more widespread, although mask wearing remained a contested policy tool. It was therefore unclear whether messaging would be similarly effective in this new context. 
We find baseline increases in scores on the BELIEFS and OWN-SD scales. Panel A of Fig. 2 plots main effects of message efficacy compared to the Control message for all outcomes (underlying regression analysis appears in Table S2). The Baseline message is associated with increased BELIEFS and OWN-SD scores ( $p<.05$, one-sided, in both cases). The Not Bravery and ORLC messages appear more effective, however: Each is associated with a statistically significant increase in four outcomes: the BELIEFS, OWNSD, OTHERS, and MASKS scales, with p-values < .05, one-sided, in all cases. The magnitudes of these effects are approximately $0.1 \mathrm{std}$. dev. for each measure. None of the messages have large or statistically precise effects on the FOOD scale.

(Fig. 2 Here)

There is less clear evidence that these messages are incrementally more effective that the baseline content to which they are added. For the BELIEFS, OWN-SD, OTHERS, and MASKS scales, both the Not Bravery and ORLC messages are associated with effects that are always larger than the baseline message, with the magnitudes of these differences ranging from $22-88 \%$ and averaging $50 \%$. Because effect sizes are still modest, however, these differences are not generally statistically distinguishable at $p$ $<.05$, two-sided, with the notable exception of the Not Bravery message which has an effect $88 \%$ larger than the Baseline message on the OTHERS scale.

Examining of the secondary outcomes plotted in Panel B of Fig. 2 shows that the messages do not have large effects on requests for mail ballots, may modestly depress a willingness to vote in person (individually not statistically significant,), and that all treatment messages increase reported willingness to get a COVID-19 vaccine when it becomes available (with all of these differences statistically significant at or near $p<.05$ ). The effect of vaccine uptake is encouraging, but finding that any message that emphasizes the threat posed by COVID-19 may depress interest in in-person voting suggests the importance of efforts to provide safe alternatives or address issues of in-person voting safety.

Differences in effects for those who endorse liberty values partially confirm Study 1 . Compared to the Control message, the Not Bravery message is more effective among those who endorse liberty for encouraging social distancing-it increases OWN-SD measure by .027 units ( $90 \% \mathrm{Cl}$ : .009, .043), an effect that is $70 \%$ larger than the effect for those who do not endorse liberty values. This difference is not significant, however, and the estimates for the other outcomes are inconsistently signed. If we instead focus on the relative effectiveness of the Not Bravery message compared to the Baseline message, a test that accounts for the fact that those who endorse liberty values may respond differently to the baseline content, we uncover more evidence that those who endorse liberty values respond more to the Not Bravery treatment. In particular, for those who endorse liberty values, the Not Bravery message is between $20 \%$ and $125 \%$ more effective than the Baseline message for the five primary outcomes. The largest difference is for the OWN-SD scale outcome, where the difference is .014 (90\% Cl: $-.004, .033)$.

In addition to our scale outcomes, we also focus on several individual items of particular interest, including the three measures of compliance with government policies to reduce the spread of COVID-19 discussed above: Self-isolation for those exposed, alerting authorities if testing positive, and cooperation 
with authorities in contact tracing. These items are included in the OWN-SD behavior index, but are also individually of interest because they are areas where governments have reported difficulty obtaining compliance. Figure 3 show that the Not Bravery message is associated with a statistically significant increase in self-isolation and willingness to alert authorities, effects that are larger than and statistically distinguishable from the effects of the baseline message. (Underlying regression results appear in Table S3) Similarly, the ORLC message is associated with a statistically significant increase in self-isolation and willingness to cooperate in contact tracing, effects that are larger than and statistically distinguishable from the effects of the baseline message.

(Fig. 3 Here)

Second, we also examine effects on three isolated behaviors, attendance at religious gatherings and inside visits to a friend and family member's house. Religious gatherings have emerged as sources of conflict over prohibitions on group meetings (18), while private indoor meetings are thought to be vehicles by which asymptomatic individuals expose those who are at more serious risk for infection. Once again, these items are individually in the OWN-SD behavior index. Results appear in Fig. 3. The Not Bravery Message is associated with statistically significant increases in all three outcomes, while the ORLC message is associated with changes in both the family and friend small gathering outcomes. The Not Bravery effect for attendance at religious services is statistically distinguishable from the effect of the Baseline message $(p<.05)$ and the ORLC effect for each type of private gatherings is also statistically larger than the effect of the baseline message $(p<.03$ and .05 , respectively).

The results presented here show that public health messaging that reframes bravery as unattractive and selfish or invokes cooperative social norms that everyone is contributing to protect others can increase behavioral intentions and beliefs about social distancing that helps reduce the spread of COVID-19. Further, this increase in short-term health promoting attitudes does not appear to come at the cost of reducing intentions to receive a vaccine in the future.

Moral foundations theory $(17,19)$, which postulates that humans have several underlying common values that are differentially emphasized by various individuals, has been used to explain health behaviors such as vaccination (20). Increasingly, opposition to public health measures is grounded in the language of personal freedoms (20) and, indeed, concerns about government infringement on personal freedoms have arisen during the COVID-19 pandemic $(21,22)$. We find that emphasis on liberty value modifies the impact of the Not Bravery treatment -indicating that such messages are particularly powerful for those for whom personal freedoms are important.

Our findings can inform both mass public health messaging initiatives (e.g. those deployed on social and electronic media) as well as interpersonal communication strategies such as healthcare provider-level communication and persuasion. Our findings regarding increases in vaccine acceptance - even when the treatment messages were focused on broader social distancing behaviors - are encouraging as current evidence points to acceptance rates of an eventual COVID-19 vaccine below the nominal herd immunity 
threshold. While this work shows robust attitude change in response to public health messaging, field tests are needed to assess the consequences for people's social distancing behavior.

\section{Methods}

\section{Experiment 1}

We recruited 2,500 respondents between May 21 and May 22, 2020 using the survey firm Lucid.io, which recruits subjects online using Census-based quota sampling ( $n=2,531$ completed the survey). After respondents provided informed consent and answered a variety of demographic questions, supplementing prior demographic information gathered by Lucid, subjects were randomly assigned to the baseline message or one of the ten different treatment messages. The ten treatment messages added additional content to the baseline message.

Given the number of comparisons to baseline message efficacy, we assigned respondents to that message with a $3 / 13$ chance and all other treatments with a chance of $1 / 13$. Respondents could not advance the survey page that displayed the treatment message until 30 seconds had elapsed. After exposure to the treatment message, subjects completed a battery of outcome measurements that were presented in a fixed order. The questions either appeared as individual questions or within grids that asked the respondent's likelihood of engaging in different behaviors. After the outcomes, we measured political attitudes, personal endorsement of the liberty moral foundation, and experience with COVID-19. Respondents then completed the survey by reading a short debrief about the importance of social distancing that included a link to the Center for Disease Control's most recent public health advice concerning COVID-19.

We analyzed the data using ordinary least squares (OLS) regression with robust (Huber/White) standard errors. Each outcome was predicted with a model that included an indicator variable for every treatment condition, excluding the baseline condition, with covariates for the respondent's age (in years), whether they were female, their household income as a scale, an indicator variable if they did not provide their household income, their level of education as indicators, their political partisanship on the standard 7point scale as indicators, and the geographic region of the United States that they are located in as indicators. For our subgroup analyses, we remove the predictor for the subgroup in the model, but keep the remaining covariates, with the exception of the liberty endorsement which was not included as a covariate in the primary analysis. Full results of each model are reported below.

Approximately $81 \%$ of respondents assigned to treatment continued to the remainder of the survey. Survey attrition was lower among those assigned to most of the treatment messages apart from the baseline message, by up to 8 percentage points. We examined whether pre-treatment covariates that predicted this attrition, as well as pre-treatment covariates that explain outcomes, differentially predict this attrition across treatments and found no evidence that they did. As a robustness test, we also examined whether imputing missing outcomes using the relationship between pre-treatment covariates 
and outcomes in the baseline condition affected our inferences. Results are somewhat attenuated with larger standard errors.

\section{Experiment 2}

The design of Experiment 2 followed the framework of Experiment 1 with differences noted here. The messages tested in Experiment 2 were the two most promising messages from Experiment 1 (Not Bravery and IOCLP), whose performance was compared both to a control group exposed to an unrelated placebo message about bird feeding and a modified version of the baseline treatment message from Experiment 1. Subjects were assigned to each of these four conditions at equal rates. As before, the novel treatment language was added to the baseline treatment.

Comparisons of treatment effectiveness to each benchmark, as well as specific tests for differences in the effectiveness of the Not Bravery message by liberty endorsement, were pre-registered at Open Science Framework with one-sided hypotheses tests in light of the evidence from Experiment 1. Given this, we measured liberty endorsement pre-treatment in the survey. Otherwise, the flow of the study was the same as Experiment 1. All scale construction and coding rules, including novel items not used in Experiment 1 , were also pre-registered.

\section{Treatment Messages}

The baseline treatment message was:

To end the COVID-19 pandemic, public health officials believe we should practice social distancing. Social distancing means that you should:

- Work from home when possible

- Wear a mask that covers your nose and mouth when outside of your home around other people

- Stay at least 6 feet away from others if you need to go out in public, for example to shop for food or medicine

- Avoid large gatherings, especially indoors

- Stay home except to seek medical care if you are sick or have recently had close contact (closer than 6 feet for at least 15 minutes) with a person with COVID-19

- Avoid pooled rides or rides where multiple passengers are picked up who are not in the same household

The Not Bravery message added this content to the baseline message:

Soldiers, fire fighters, EMTs, and doctors are putting their lives on the line to serve others during the COVID-19 pandemic. That's bravery. 
But people who don't practice social distancing because they don't think COVID is a big deal or don't think they will get sick aren't being brave, they are reckless. By not social distancing, you are risking the health of your family, friends, and community.

There is nothing attractive and independent-minded about ignoring public health guidance to practice social distancing. Not social distancing means you risk the health of others. To show strength practice social distancing so you don't get sick and take resources from other people who need them more, or risk spreading the disease to those who are at risk. Social distancing may be inconvenient, but it works.

The IOCLP message added this content to the baseline message:

Stopping COVID-19 is important because it reduces the risk that members of your family and community could get sick and die. COVID-19 kills people of all ages. Even for those who are young and healthy, there is a risk of death or long-term disability. Many other people are already social distancing. Remember, every person who practices social distancing reduces the risk that people you care about get sick. While you can't do it alone, we can all protect everyone by working together.

Power calculations indicated greater than $80 \%$ power to detect treatment effects $75 \%$ as large as in Experiment 1 with an $\mathrm{N}$ of 3000 assuming scale distributions were the same as observed in Experiment 1. This study was fielded using the vendor YouGov, which recruits subjects from its existing online panel to match Census and American Community Survey benchmarks and additionally provides weights to account for any remaining differences between target and realized samples. Experiment 2 was fielded in two waves, the first was between July 15 and July 21, $2020(\mathrm{~N}=3000)$ and the second was between July 31 and August 10, 2020 ( $\mathrm{N}=3079)$. The study was fielded twice because of an implementation error in programming by the vendor survey content that followed the items analyzed here for the first fielding (the error was for items for an unrelated project that was not about COVID-19, and which followed all of the items analyzed here), causing the vendor to re-field the entire survey. Our final $\mathrm{N}$ is therefore approximately twice as large as the sample described in our pre-registration document ( $\mathrm{N}=3000$ preregistered, $\mathrm{N}=6079$ in final analysis dataset).

Our estimation strategy for Experiment 2 was the same as Experiment 1. The primary analysis included all of the covariates described in Experiment 1 as well as race and a four-category item that measured a respondent's employment status (working at home, working outside the home by choice, working outside the home because it required, or not working). We also estimate effects without pre-treatment covariates and with weights provided YouGov. Further, we report subgroup analyses by liberty endorsement, partisanship, gender, and employment status. Again, estimates for all models are provided below. We do not have data for individuals who did not complete the entire survey in this fielding.

The design of Experiment 2 followed the framework of Experiment 1 with differences noted here. The messages tested in Experiment 2 were the two most promising messages from Experiment 1 (Not Bravery and IOCLP), whose performance was compared both to a control group exposed to an unrelated placebo message about bird feeding and a modified version of the baseline treatment message from Experiment 
1. Subjects were assigned to each of these four conditions at equal rates. As before, the novel treatment language was added to the baseline treatment.

Comparisons of treatment effectiveness to each benchmark, as well as specific tests for differences in the effectiveness of the Not Bravery message by liberty endorsement, were pre-registered at Open Science Framework with one-sided hypotheses tests in light of the evidence from Experiment 1. Given this, we measured liberty endorsement pre-treatment in the survey. Otherwise, the flow of the study was the same as Experiment 1. All scale construction and coding rules, including novel items not used in Experiment 1 , were also pre-registered.

\section{Data Availability}

Raw data and code for all analyses will be made available at Harvard Dataverse upon publication of this work.

\section{Ethical Oversight}

Both experiments were fielded under an IRB exemption granted by a university IRB.

\section{References}

1. M. Stead, K. Angus, T. Langley, et al., Mass Media to Communicate Public Health Messages in Six Health Topic Areas: A Systematic Review and Other Reviews of Evidence (NIHR Journals Library, Southampton, UK, 2019).

2. A. I. Abioye, K. Hajifathalian, G. Danaei, Do mass media campaigns improve physical activity? A systematic review and meta-analysis. Archives of Public Health 71, 20 (2013)

3. T. Halkjelsvik, Do disgusting and fearful anti-smoking advertisements increase or decrease support for tobacco control policies? International Journal of Drug Policy 25, 744-747 (2014).

4. R.G. Jepson, F.M. Harris, S. Platt, C. Tannahill, The effectiveness of interventions to change six health behaviours: a review of reviews. BMC Public Health 10<bi <,</bi> 538 (2010).

5. I.S. Speizer, R.J. Magnani, C.E. Colvin, The effectiveness of adolescent reproductive health interventions in developing countries: a review of the evidence. $J$ Adolesc Health $33,324-348$ (2003).

6. G.W. Farthing, Attitudes toward heroic and nonheroic physical risk takers as mates and as friends. Evolution and Human Behavior 26, 171-185 (2005).

7. S. Kelly, R.I.M. Dunbar, Who dares, wins. Human Nature 2, 89-105 (2001).

8. A. Wilke, J.M. Hutchinson, P.M. Todd, D.J. Kruger. Is risk taking used as a cue in mate choice? Evolutionary Psychology 4, 367-393 (2006).

9. C. Betsch, R. Böhm, L. Korn, C. Holtmann, On the benefits of explaining herd immunity in vaccine advocacy. Nature Human Behaviour 1, 0056 (2017). 
10. J. Jordan, E. Yoeli, D. Rand, https://psyarxiv.com/yuq7x/ (2020).

11. J.T. Vietri, M. Li, A.P. Galvani, G.B. Chapman, Vaccinating to help ourselves and others. Medical Decision Making, 32, 447-458 (2012).

12. A.W. Delton, L. Cosmides, M. Guemo, T.E. Robertson, J. Tooby, The psychosemantics of free riding: Dissecting the architecture of a moral concept. Journal of Personality and Social Psychology 102, 1252-1270 (2012).

13. D. Centola, An experimental study of homophily in the adoption of health behavior. Science 334, 1269-1272 (2011).

14. E. Fehr, U Fischbacher, Social norms and human cooperation. Trends in Cognitive Sciences 8, 185190 (2004).

15. R.R. Reno, R.B. Cialdini, C.A. Kallgren, The transsituational influence of social norms. Journal of Personality and Social Psychology, 64, 104-112 (1993).

16. J.J. Van Bavel, Using social and behavioural science to support COVID-19 pandemic response. Nature Human Behaviour 4, 460-471 (2020).

17. R. Iyer, S. Koleva, J. Graham, P. Ditto, J. Haidt, Understanding libertarian morality: The psychological dispositions of self-identified libertarians. PloS One 7, e42366 (2012).

18. K. Conger, J. Healy, L. Tompkins, Churches were eager to reopen. Now they are confronting coronavirus cases. New York Times (2020). https://www.nytimes.com/2020/07/08/us/coronaviruschurches-outbreaks.html

19. J. Graham et al., Moral foundations theory: The pragmatic validity of moral pluralism. Advances in Experimental Social Psychology 47, 55-130 (2013).

20. A.B. Amin et al., Association of moral values with vaccine hesitancy. Nature Human Behavior 1, 873880 (2017).

21. L.O. Gosten, J.G. Hodge, US emergency legal responses to novel coronavirus: Balancing public health and civil liberties. JAMA 12, 1131-1132 (2020).

22. W.E. Parmet, M.S. Sinha, COVID-19-the law and limits of quarantine. New England Journal of Medicine 382, e28 (2020).

\section{Figures}



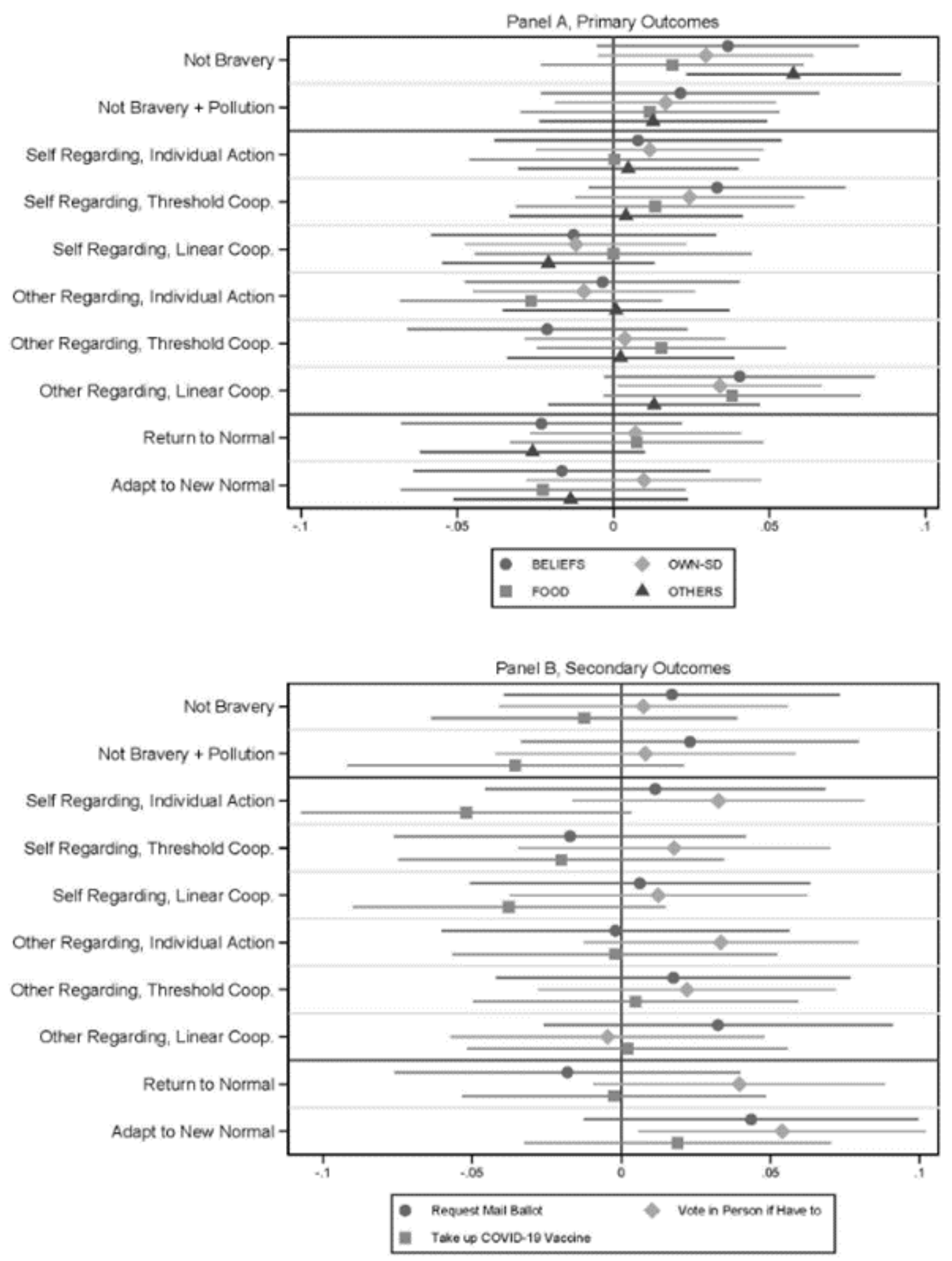

\section{Figure 1}

Experiment 1 results. The Not Bravery and ORLC messages increase beliefs about and reported intentions to practice social distancing without unintended consequences. Panel A shows OLS regression coefficient estimates for each primary outcome by treatment compared to the baseline control. Panel B shows the same information for the secondary outcomes. Both panels display $95 \%$ confidence intervals. 

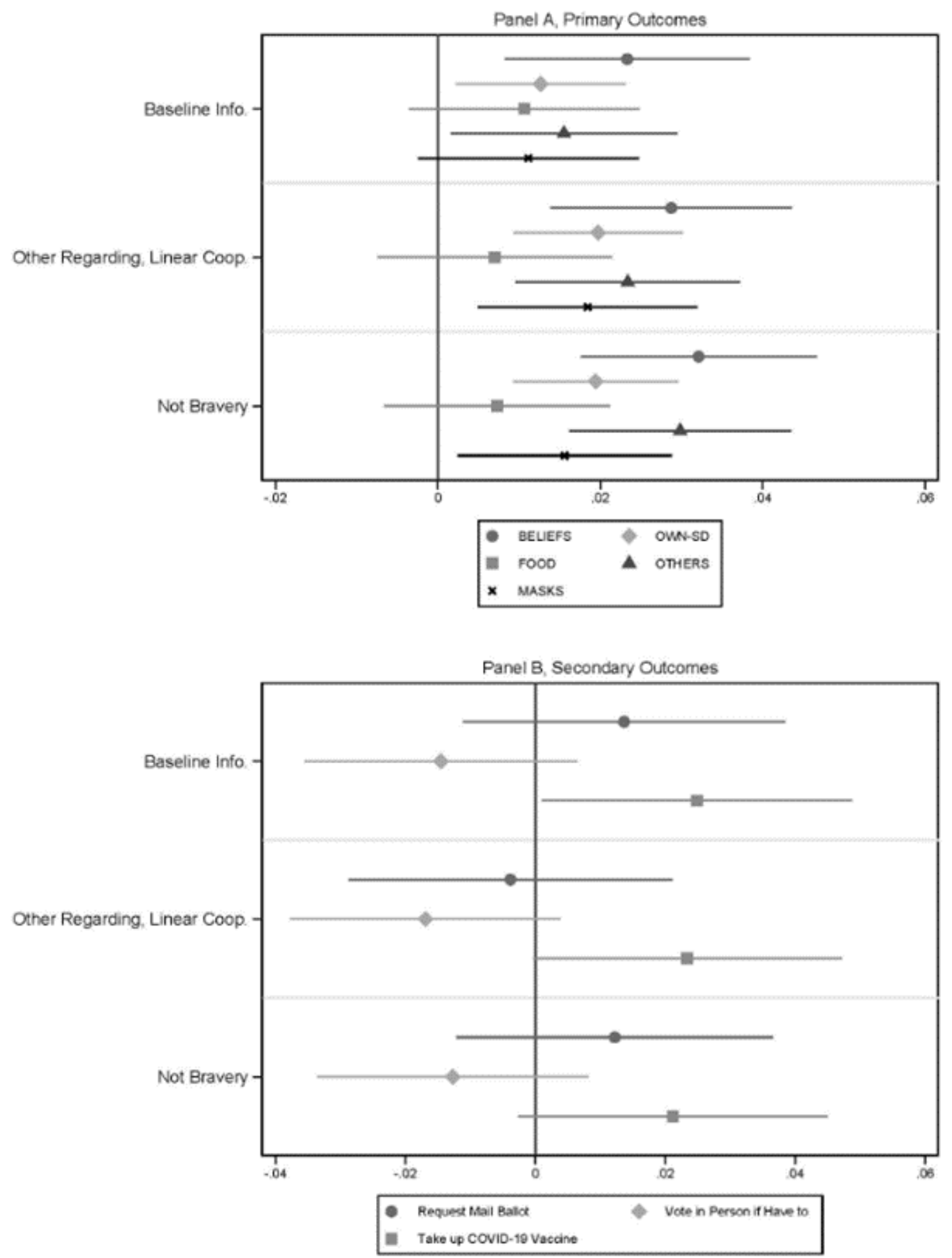

\section{Figure 2}

Experiment 2 results. Compared to the placebo control, the baseline message, the Not Bravery message, and the ORLC increase beliefs and reported behavioral intentions to practice social distancing. Panel A shows OLS regression coefficient estimates for each primary outcome by treatment compared to the placebo control. Panel B shows the same information for the secondary outcomes. Both panels display $90 \%$ confidence intervals. 


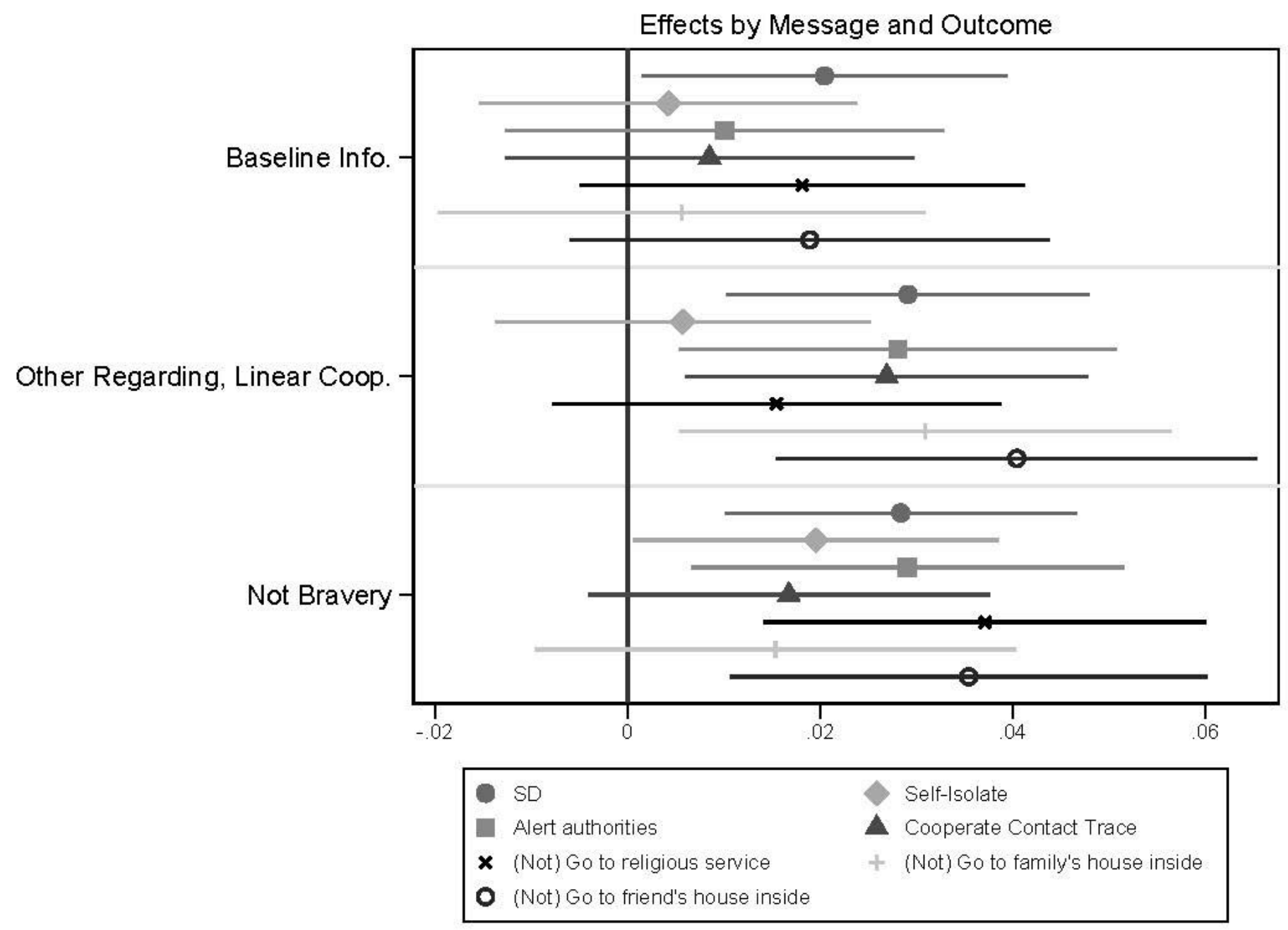

Figure 3

Experiment 3 individual social distancing items. The Not Bravery and ORLC message increase respondents reported intentions to not engage in key behaviors to reduce the spread of COVID-19 and to cooperate with government officials, even compared to the Baseline Information message. This figure shows OLS regression coefficient estimates compared to the placebo-treated control message with $95 \%$ confidence intervals.

\section{Supplementary Files}

This is a list of supplementary files associated with this preprint. Click to download.

- COVID19persuasionNatureHumanBehaviorAppendixSubmission.docx 